\title{
Analysis of model for control of thermal energy in buildings
}

\author{
Sina Orangi, Zahir Barahmand, Ashish Bhattarai, Bernt Lie \\ University of South-Eastern Norway, Porsgrunn, Norway, Bernt. Lie@usn. no
}

\begin{abstract}
The paper considers a model for floor heating in buildings, with an electrically heated, stratified water tank, a circulation loop, a detailed description of heat transport through the floor, and a simplistic room model. The model structure is suitable for control of room temperature, and the paper discusses the use of modern simulation tools for control-relevant analysis of such models. The stratification description contains a non-differentiable buoyancy term, and two approximations are studied which circumvent this problem. The results indicate that a boundary layer approach is superior to a log-sum-exp approximation. Important basic control-relevant analysis ideas include step response (time constants), sensitivity analysis (parameter identifiability), the system zeros location (attainable performance), and Bode plots (control synthesis). The paper illustrates how such an analysis can be carried out using tools such as OpenModelica in combination with scripting language Julia.

Keywords: thermal building model, heated water tank, floor heating, model analysis, sensitivity analysis, linearization, control architecture
\end{abstract}

\section{Introduction}

Modern floor heating systems utilize "low quality" thermal energy in lukewarm water $\left(30-35^{\circ} \mathrm{C}\right)$. To reduce the overall energy consumption, it is necessary to allow for rapid temperature changes when occupants leave or arrive, hence a low heat capacity in the distribution system is desirable. Maximizing the temperature in the buildings requires low heat transfer coefficients. Building energy management systems (BEMS) are used to handle the heating and water distribution.

(Lie et al., 2014) discussed the use of solar heating assisted by electric heating for floor heating, and studied the use of Model Predictive Control (MPC). In (Johansen et al., 2019), an improved model of an electric heater was considered, and compared with experimental data. Specifically, a model of stratification due to (Viskanta et al., 1977) was introduced, see also (Xu et al., 2014). A more complex model of stratification is given in (Vrettos, 2016), with a two stage diffusion predictor and buoyancy corrector step. In (Bhattarai et al., 2020), a minor correction of the buoyancy term is introduced in reference to (Johansen et al., 2019), and the system is extended to include a circulation loop for floor heating - with more details about the floor layers than in (Lie et al., 2014), while excluding the solar heating.

Traditional heating systems for buildings often use simple temperature controllers such as thermostats. It is of interest to also consider and analyze building models from a more classical control theoretical point of view. Open loop studies typically include step response tests. Interesting questions arise related to identifiability of model parameters, where parameter sensitivity plays a key role, (Varma et al., 1999), (Jayakumar et al., 2011), (Sarmiento Ferrero et al., 2006). Model structure and input-output relations may put restrictions on attainable performance in dynamic systems. Essentially, unstable zero dynamics limits how fast feedback control loops can be made while retaining robustness. For linear models, the modes of the zero dynamics equal the system zeros, (Kwakernaak and Sivan, 1972), (Lie, 1995), and the performance limiting zeros are the right-half-plane zeros. Classical linear control design is often based on linearized approximations of models, and the Bode plot is a simple presentation of some dynamic properties for importance in control design, (Åstrøm and Murray, 2008).

The emphasis of this paper is on how to use modern computer languages to analyze the model, with the ultimate purpose of control design. We consider the languages Julia (Rackauckas and Nie, 2017) and Modelica (Fritzson, 2015), specifically OpenModelica operated via the OMJulia API (Lie et al., 2019). The focus is not on control design, but on analysis tools. The floor heating model in (Bhattarai et al., 2020) is used in the study. This model contains a max () function in the description of stratification, which is unfortunate when linear approximations are sought. Thus, two approximations to the max function are considered. Basic step responses are used to find the open loop response. Next, computation of output sensitivity to model parameters is illustrated, together with model linearization with location of poles and zeros as well as Bode plots. The paper is organized as follows. In Section 2, the system under study is presented, and two approaches to model approximation are presented. In Section 3 , the original stratification model is compared to the two approximations. In Section 4, computer tools are used for model analysis. In Section 5, some conclusions are drawn.

\section{System overview}

\subsection{Floor heating}

Consider a floor heating system for a building, Fig. 1. 


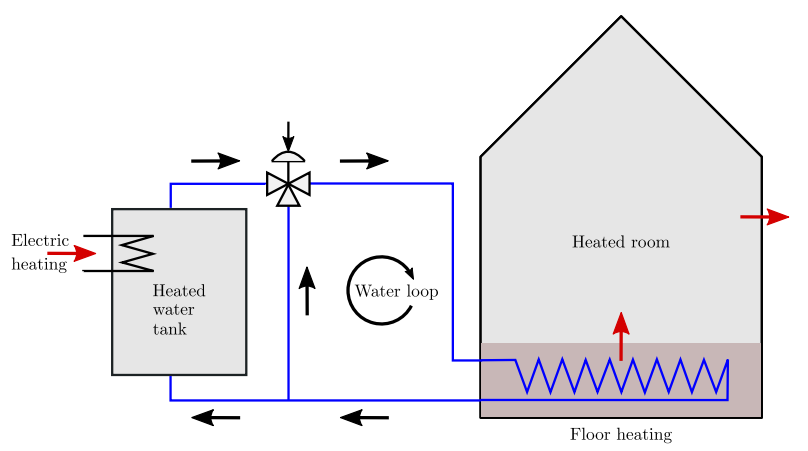

Figure 1. Floor heating system.

The system consists of an electrically heated, stratified water tank which supplies heated water to a water loop passing through water pipes embedded in the floor. The heated floor then provides heating to the room above to compensate for heat loss to the surroundings. Both floor temperature and air temperature in the heated room is of importance for inhabitant comfort. Typically, a floor temperature of ca. $22^{\circ} \mathrm{C}$ and an air temperature of ca. $20^{\circ} \mathrm{C}$ is deemed optimal when in use.

Modern buildings are not in use all the time, and it is of interest to save energy by reducing temperatures when a building is empty. To allow for quick reduction and increase in temperatures, the heat capacity of the floor should be low. On the other hand, low heat capacity leads to problems in case of power failure.

The heated water tank is influenced by external signals in the form of the loop volumetric water flow rate $\dot{V}_{\ell}$, the split range valve signal $u_{\mathrm{v}}$ which determines how much water goes through the heated tank, the ambient temperature of the heated tank, $T_{\mathrm{a}}^{\mathrm{t}}$, and the fraction of full electric power $u_{\mathrm{P}}$ that is used to heat the tank. In the model of (Johansen et al., 2019), the inlet temperature $T_{\mathrm{i}}$ at the bottom of the tank was also an external signal; in this work, $T_{\mathrm{i}}$ is a signal that comes from the heated floor subsystem, and is thus not a free, external input.

The water from the heated tank flows into the water loop at temperature $T_{\mathrm{i}}^{\ell}$ (the temperature after the split range valve of the heated tank), and passes through a lengthy pipe embedded in the floor. The floor pipe is essentially a heat exchanger for transfer of heat to the floor. From the floor, heat is transferred to the room by convection and radiation. Finally, the room experiences a heat loss to the surroundings which is at external ambient temperature $T_{\mathrm{a}}^{\mathrm{r}}$.

\subsection{Buoyancy conductivity approximations}

\subsubsection{Original stratification expression}

The buoyancy conductivity expression $k_{\mathrm{b}}$ is

$$
k_{\mathrm{b}} \propto \sqrt{\max \left(-\frac{\partial T}{\partial z}, 0\right)},
$$

or alternatively

$$
k_{\mathrm{b}} \propto \begin{cases}\sqrt{-\frac{\partial T}{\partial z}}, & \frac{\partial T}{\partial z}<0 \\ 0, & \frac{\partial T}{\partial z} \geq 0 .\end{cases}
$$

For implementation in Modelica, the formulation in Eq. 1 works fine, while the formulation in Eq. 2 leads to chattering between the two function branches when the gradient fluctuates around 0 .

If we want to linearize the model in Modelica, things get even more complex: Modelica does not handle automatic linearization of either the expression in Eq. 2 or that in Eq. 1. Thus, for analysis purposes, it is of interest to seek approximations to the above buoyancy conductivity expressions which allow for linearizing the model by automatic linearization.

\subsubsection{Log-sum-exp approximation}

For simplicity, we first consider function

$$
\max (-x, 0)= \begin{cases}-x, & x<0 \\ 0, & x \geq 0\end{cases}
$$

A common approximation of this max function is the socalled "log-sum-exp" function, (Lago et al., 2019), which in a simple version can be posed as

$$
\begin{aligned}
\max (-x, 0) & \approx \frac{1}{\mu} \log (\exp (-\mu x)+\exp (\mu \cdot 0)) \\
& =\frac{1}{\mu} \log (1+\exp (-\mu x)) .
\end{aligned}
$$

With increasing $\mu$, this approximation becomes better and better, while still being differentiable. Thus, we can use the following approximation of the buoyancy conductivity:

$$
k_{\mathrm{b}} \propto \sqrt{\frac{1}{\mu} \log \left(1+\exp \left(-\mu \frac{\partial T}{\partial z}\right)\right)},
$$

where $\mu$ is tuned to give as good approximation as possible.

There is a potential problem with this approximation for our use: $\frac{1}{\mu} \log (1+\exp (-\mu x))>0$ for $x>0$. Thus, when $\frac{\partial T}{\partial z} \rightarrow x$, we will get a "buoyancy" effect when $\frac{\partial T}{\partial x}>0-$ when there really is no buoyancy.

\subsubsection{Boundary layer approximation}

An alternative approach to approximation is to establish a boundary layer for $x \in[-\delta, 0]$, and use a polynomial transition from $y(-\delta)$ to $y(0)$ which simultaneously is differentiable at $x=-\delta$ and $x=0$. For simplicity, we approximate $\sqrt{\max (-x, 0)}$ instead of $\max (-x, 0)$. It can be found that the following composed function is suitable:

$$
\sqrt{\max (-x, 0)} \approx \begin{cases}\sqrt{-x}, & x<-\delta \\ \sqrt{\delta} \cdot\left(\frac{3}{2}\left(\frac{x}{\delta}\right)^{3}+\frac{5}{2}\left(\frac{x}{\delta}\right)^{2}\right), & x \in[-\delta, 0] \\ 0, & x>0 .\end{cases}
$$



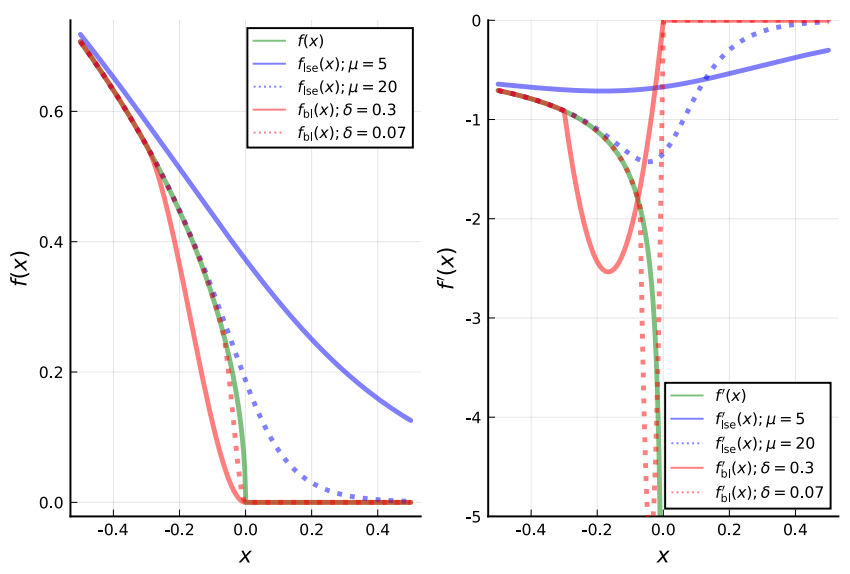

Figure 2. Left panel: comparison of $f(x)=\sqrt{\max (-x, 0)}$ (green line) with log-sum-exp approximation $f_{\text {lse }}(x)=$ $\sqrt{\frac{\log (1+\exp (-\mu x))}{\mu}}$ in Eq. 3 (blue, solid: $\mu=5$, dotted: $\mu=20$ ), vs. boundary layer approximation $f_{\mathrm{bl}}(x)$ in Eq. 4 (red, solid: $\delta=0.3$, dotted: $\delta=0.07$ ). Right panel: comparison of derivatives.

\subsubsection{Comparison of approximations}

Figure 2 illustrates the approximation of $\sqrt{\max (-x, 0)}$ with these two approaches.

Figure 2 illustrates a potential problem with the logsum-exp approximation in Eq. 3 in that this approximation will give a buoyancy effect when $\frac{\partial T}{\partial z}>0$ (function $f_{\text {lse }}(x)$, blue curve in the left panel Fig. 2 is positive for $x>0$ ), which is unphysical. The boundary layer approximation in Eq. 4 (function $f_{\mathrm{bl}}(x)$, red curve in the left panel) is, however, zero for $x>0$, which is physically correct.

It should be observed that the log-sum-exp approximation gives a better approximation with $\mu \gg 5$, but OpenModelica fails to linearize the model when $\mu \gtrsim 5$.

\subsection{Transport delay in heating loop}

The advection term related to flow of water in the loop of the heater and the floor, has been approximated by a Padé approximation as in (Bhattarai et al., 2020):

$$
\begin{aligned}
\frac{T(s ; x=L)}{T(s ; x=0)} & =\frac{1}{\exp \left(\tau_{N} s\right)} \prod_{i=1}^{N-1} \frac{\exp \left(-\frac{\tau_{i}}{2} s\right)}{\exp \left(\frac{\tau_{i}}{2} s\right)} \\
& \approx \frac{1}{1+\tau_{N} s} \prod_{i=1}^{N-1} \frac{1-\frac{\tau_{i}}{2} s}{1+\frac{\tau_{i}}{2} s} .
\end{aligned}
$$

The advantage of including the lag term $\frac{1}{1+\tau_{N} S}$ is that this removes the need to differentiate the input signal $T(t ; x=0)$.

\section{Simulation with buoyancy approxi- mations}

The heated tank model from (Johansen et al., 2019) has been corrected as discussed (Bhattarai et al., 2020). Here, we study the accuracy of the log-sum-exp approximation

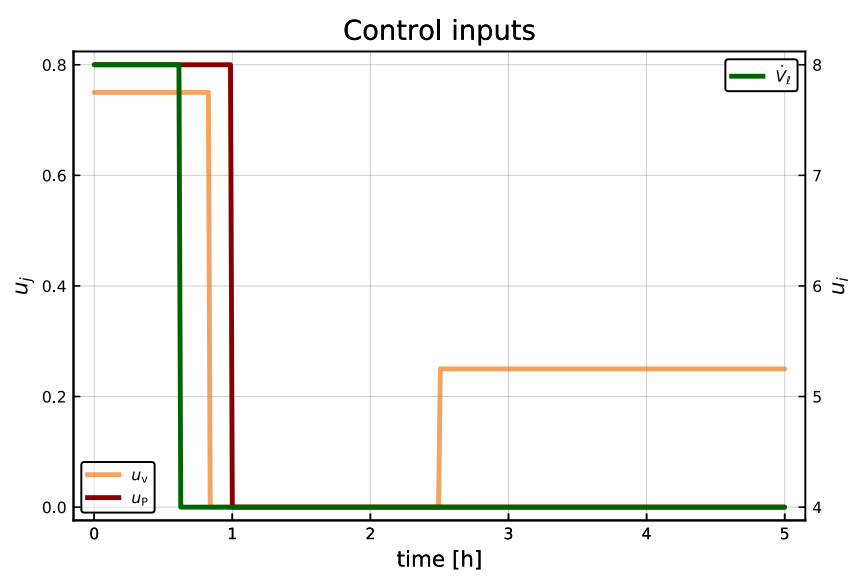

Figure 3. Control inputs $u_{\mathrm{P}}$ (power fraction to heated tank), $u_{\mathrm{v}}$ (water flow valve opening), and $\dot{V}_{\ell}$ (volumetric flow rate in heating loop).

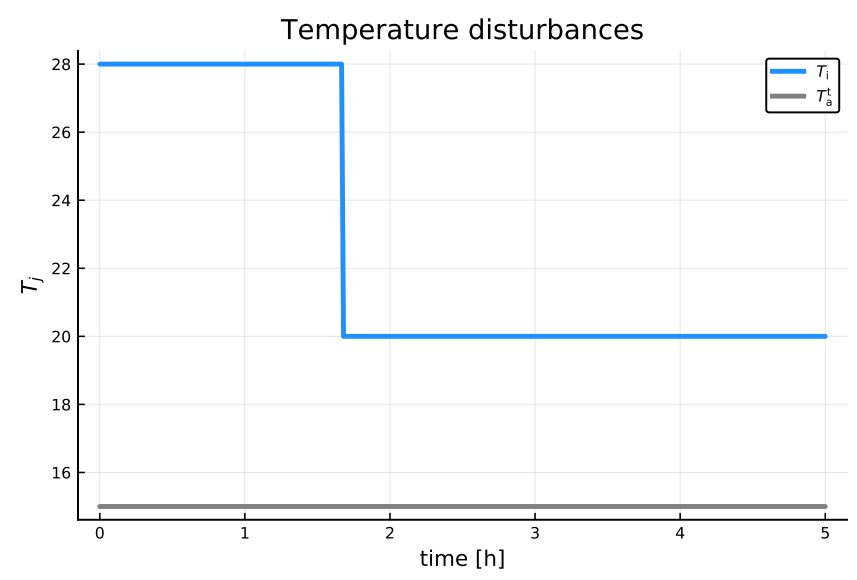

Figure 4. Disturbance temperature inputs $T_{\mathrm{i}}$ (influent to heated tank) and $T_{\mathrm{a}}^{\mathrm{t}}$ (ambient to tank).

and the boundary layer approximation introduced in Section 2.2, when applied to the heated tank. Next, we study the effect of the approximations on a combined heater and floor heating loop, as discussed in (Bhattarai et al., 2020). In all cases, stratification models with 20 discretization layers are used in the heated tank models.

\subsection{Heated tank}

The following input signals are used for the heated tank: Fig. 3 depicts the power input $u_{\mathrm{P}}$, the valve input $u_{\mathrm{v}}$, and the volumetric loop flow rate $\dot{V}_{\ell}$.

Figure 4 shows the input temperature $T_{\mathrm{i}}$ to the heated tank, and the ambient temperature surrounding the heater, $T_{\mathrm{a}}^{\mathrm{t}}$.

The temperature distribution in the heated tank using the corrected expression for buoyancy conduction compared to (Johansen et al., 2019), with approximations, is shown in Fig. 5 when using OpenModelica with solver DASSL. In Fig. 5, observe in particular that the log-sumexp approximation gives a different solution for some internal heated tank locations, see ellipses I and II in the figure. 


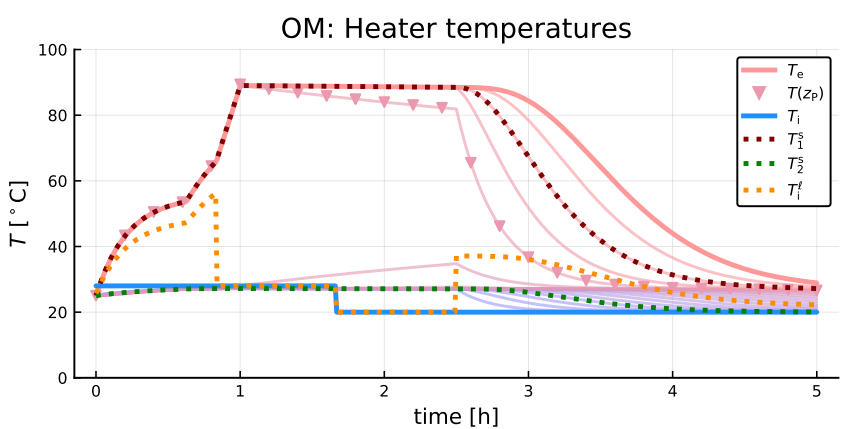

OM: Heater temperatures - log-sum-exp approximation
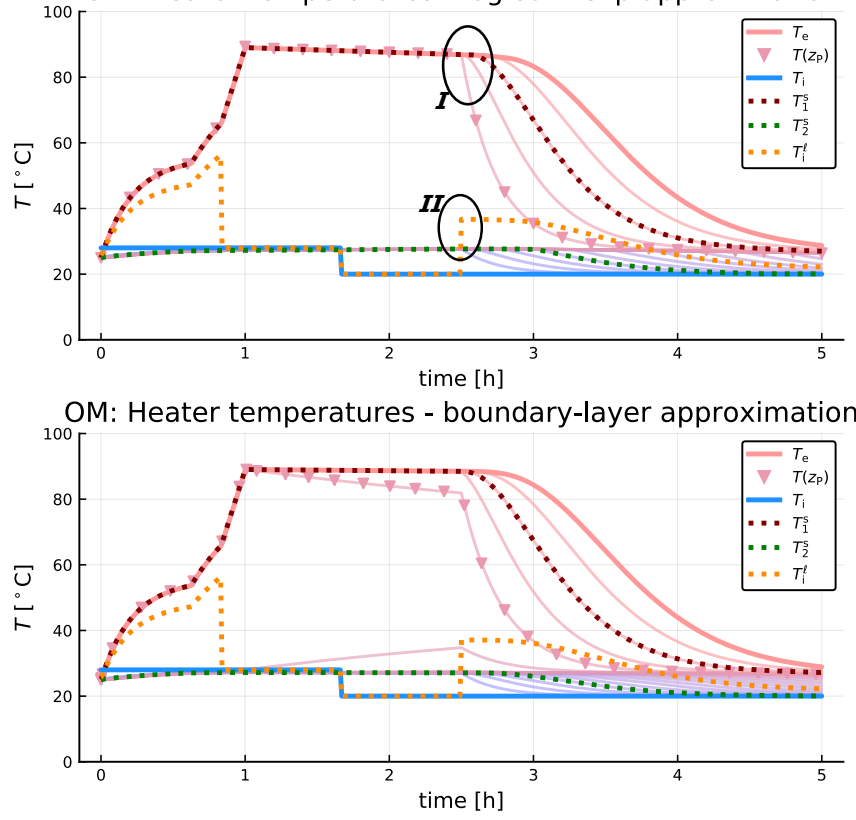

Figure 5. Temperature distribution $\left(T_{\mathrm{e}}\right.$ : effluent temperture from heated tank; $T\left(z_{\mathrm{P}}\right)$ : temperature at heating element; $T_{\mathrm{i}}$ : influent temperature to heated tank; $T_{j}^{\mathrm{s}}$ temperature at heated tank sensors; $T_{\mathrm{i}}^{\ell}$ inlet temperature to floor heating loop) in tank with corrected buoyancy conduction + approximations. Model implemented in Modelica, solved via OMJulia using OpenModelica with default solver DASSL.

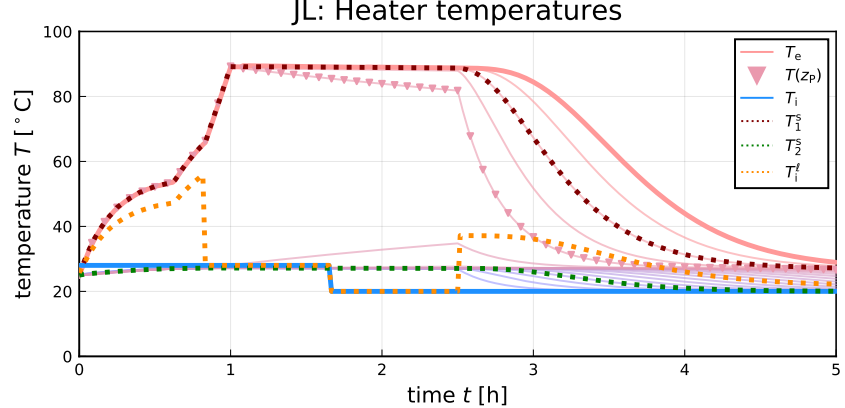

JL: Heater temperatures - log-sum-exp approximation
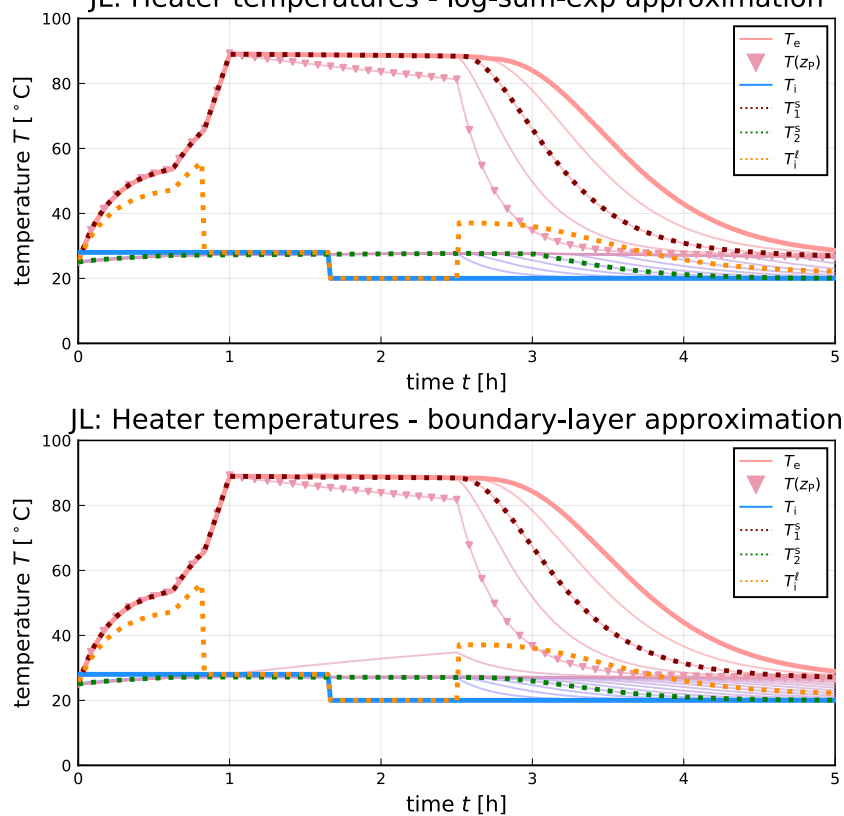

Figure 6. Temperature distribution in tank with corrected buoyancy conduction + approximations. Model implemented in Julia, solved via package DifferentialEquations with solver CVODE_Adams from the Sundials package.

The similar simulations in Julia are depicted in Fig. 6. It turns out that most Julia DifferentialEquations solvers struggled with solving the log-sum-exp approximation, reporting that the system is unstable. The CVODE solvers of the Sundials package handles the problem, though, and the CVODE_Adams solver appears to give the solution closest to the original formulation with the max function. Observe that the log-sum-exp approximation in Julia avoids the problem associated with ellipse I of Fig. 5, but retains the problem of ellipse II of the OpenModelica solution. However, for Julia, the expression $\log (1+x)$ can be replaced by an improved function $\log 1 p(x)$ when $\mathrm{x}$ is small. When doing so, the problem of ellipse II is also removed, and the log-sum-exp approximation can be made virtually indistinguishable from the solution of the max function in Julia. Still, both the original max formulation and boundary layer approximation allows for the use of standard solvers, while the log-exp-sum approximation requires Sundials solvers to find the solution.

Both when using the OpenModelica solver and the Julia solver, the boundary layer approximation works without 

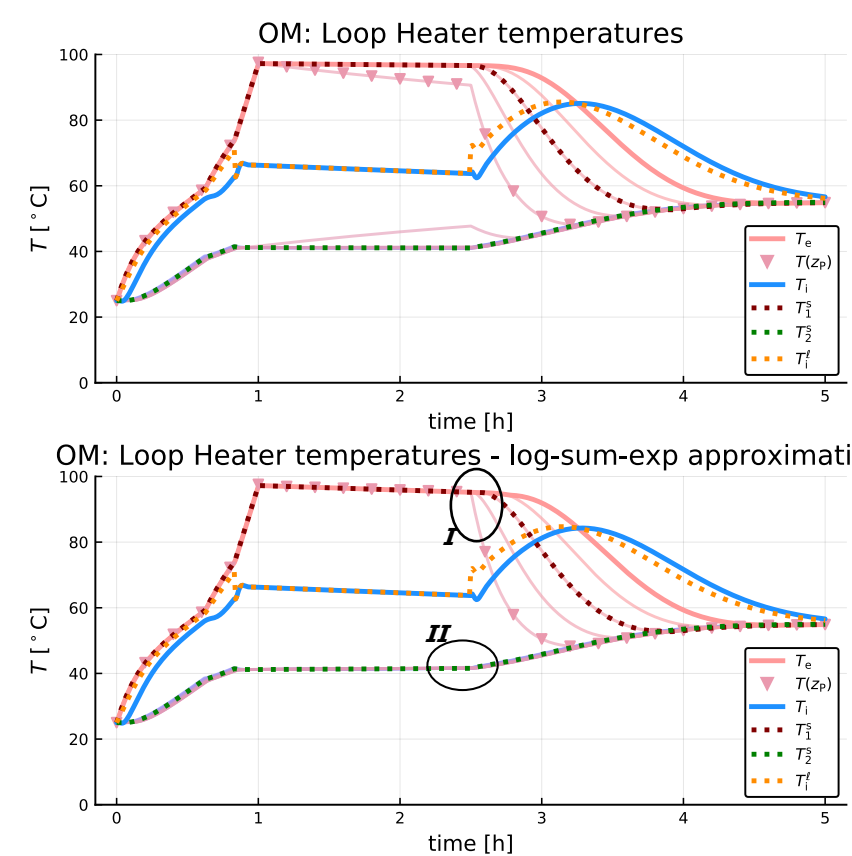

OM: Loop Heater temperatures - boundary layer approximat

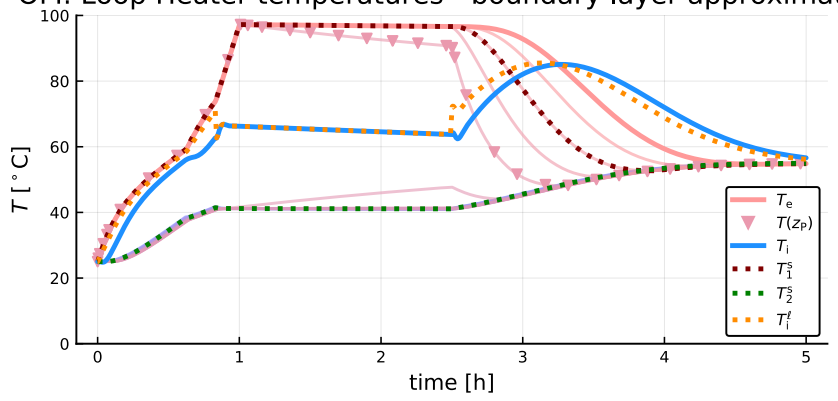

Figure 7. Temperature distribution in tank with floor loop and with corrected buoyancy conduction + approximations. Model implemented in Modelica, solved via OMJulia using OpenModelica with default solver DASSL.

problems, and gives a solution very similar to the original solution. In summary, the results indicate that the logsum-exp approximation is numerically challenging, even when using such a "poor" value of $\mu$ as $\mu=5$, see Fig. 2 .

\subsection{Heated tank + floor heating loop}

Next, we consider the combined heated tank and floor heating loop. The inputs are as in Figs. 3-4, except that the input temperature $T_{\mathrm{i}}$ (Fig. 4) to the heated tank now is a state, and is computed from the model.

The temperature distribution in the heated tank with floor loop using the corrected expression for buoyancy conduction compared to (Johansen et al., 2019) as well as approximations, are shown in Fig. 7.

Again, Fig. 7 indicates some numeric problem with the log-sum-exp approximation, see ellipses I and II.

It is also of interest to see whether there are differences in the floor-room temperatures. The temperatures in the floor layers and room of the tank with floor loop (original buoyancy expression and approximations) are shown in Fig. 8.

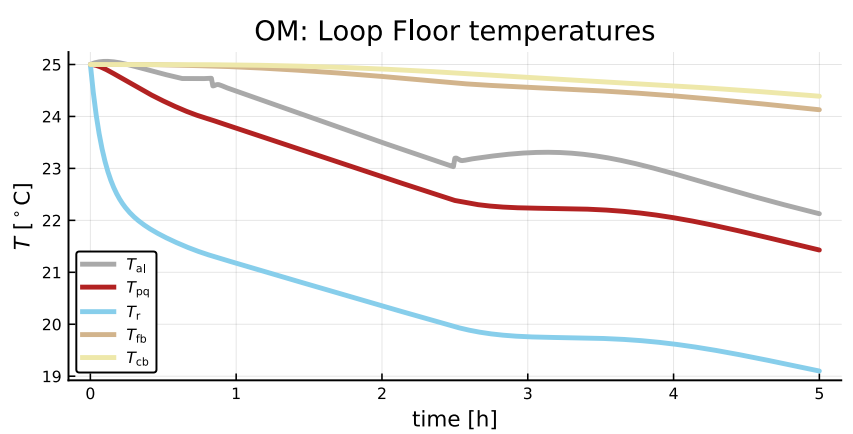

OM: Loop Floor temperatures - log-sum-exp approximatior

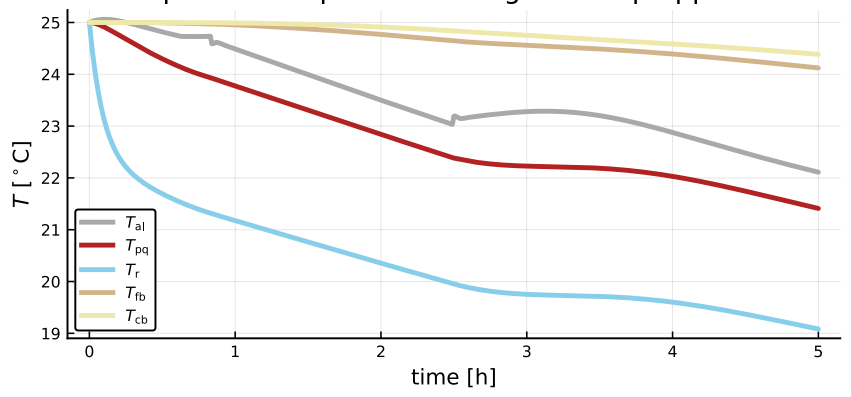

OM: Loop Floor temperatures - boundary layer approximatic

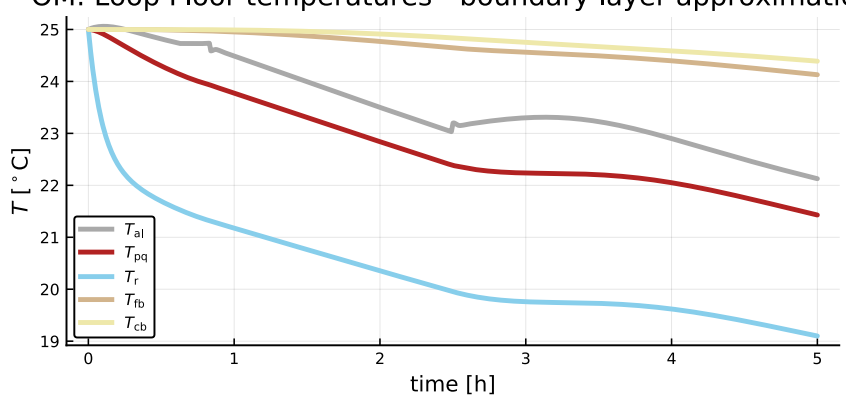

Figure 8. Temperature distribution in floor layers and room of heated tank with floor loop ( $T_{j}$ : temperature in compartment $j$ - al: aluminum plate, pq: parquet, r: room, fb: fiber board, cb: chip board). Model implemented in Modelica, solved via OMJulia using OpenModelica with default solver DASSL. 

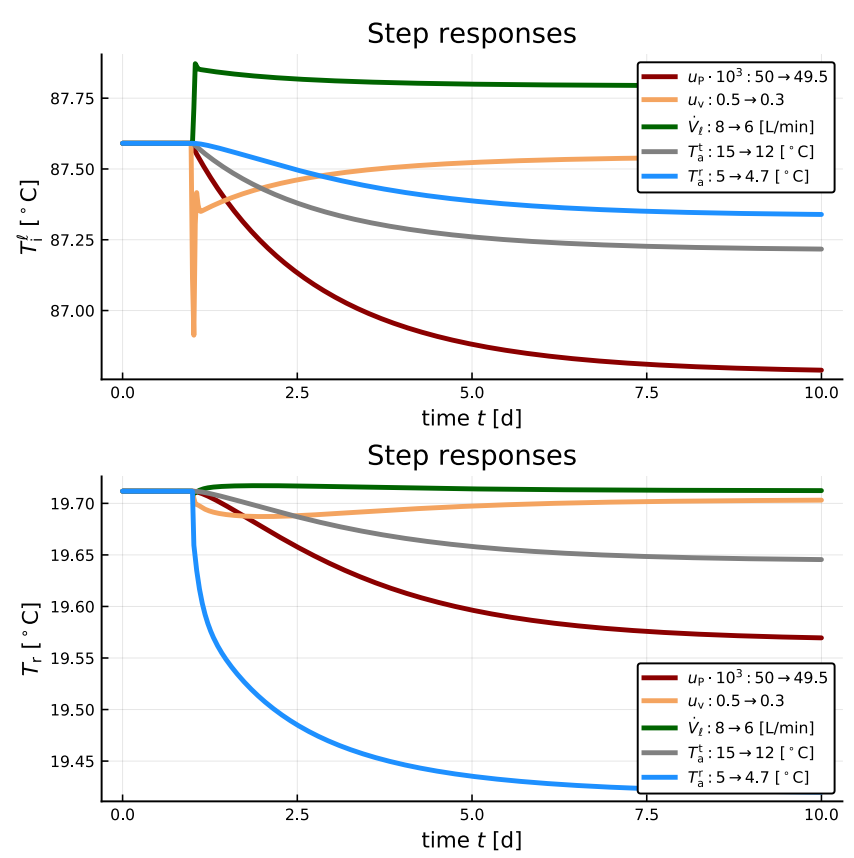

Figure 9. Step responses in $T_{\mathrm{i}}^{\ell}$ (upper panel) and $T_{\mathrm{r}}$ (lower panel) to changes in inputs $\left(u_{\mathrm{P}}\right.$ : power fraction in heater, $u_{\mathrm{V}}$ : heater bypass fraction, $\dot{V}_{\ell}$ : loop volumetric flow rate, $T_{\mathrm{a}}^{\mathrm{t}}$ : ambient temperature for heated tank, $T_{\mathrm{a}}^{\mathrm{r}}$ : ambient temperature for room, i.e., outdoor temperature), starting at steady state.

As seen from Fig. 8, the temperatures in the floor layers and the room are practically the same, independently of the buoyancy approximation.

\section{Model analysis}

\subsection{Step response}

The response in an output to a step change in an input, starting from steady state, gives a good idea of time constants in the system. Figure 9 shows the responses in temperatures $T_{\mathrm{i}}^{\ell}$ and $T_{\mathrm{r}}$ to step changes in inputs.

As seen Fig. 9, a step change in the heater by-pass fraction $\left(u_{\mathrm{v}}\right)$ gives an inverse response in both the loop inlet temperature $\left(T_{\mathrm{i}}^{\ell}\right)$ and the room temperature $\left(T_{\mathrm{r}}\right)$. Inverse response is intimately related to right half plate (RHP) zeros, and indicate a limitation in how fast the system can be controlled using this control signal. A similar inverse response may be seen in the response to $\dot{V}_{\ell}$, while the response to $u_{\mathrm{P}}$ does not have inverse response. Based on these observations, $u_{\mathrm{P}}$ is probably the best choice of con- ${ }_{1}$ trol signal.

Step changes in disturbances $T_{\mathrm{a}}^{\mathrm{t}}$ and $T_{\mathrm{a}}^{\mathrm{r}}$ are related to similar limitations in observers/state estimators, but in that case, the assessment is more complicated.

\subsection{Parameter sensitivity}

Sensitivity in output $y$ to a parameter $\theta$ is defined as $\frac{\partial y}{\partial \theta}$, and is informative as to what degree an infinitesimal change in $\theta$ will change the output $y$. If $\frac{\partial y}{\partial \theta} \equiv 0$, this implies that changing parameter $\theta$ will not change the model
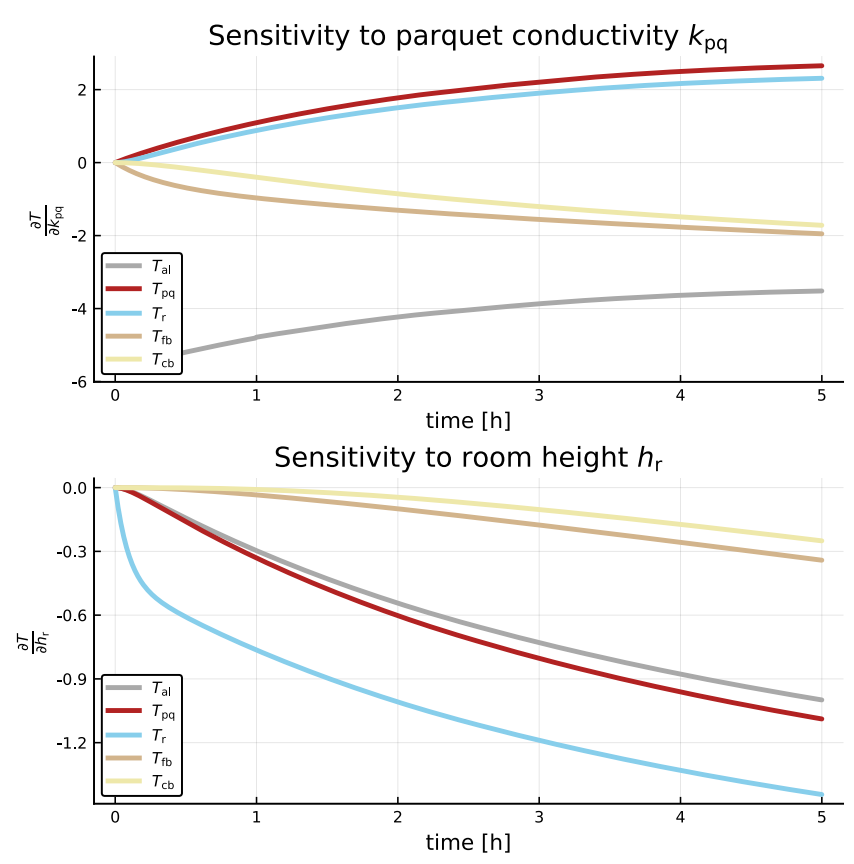

Figure 10. Sensitivities in floor-room temperatures to infinitesimal changes in parquet conductivity $k_{\mathrm{pq}}$ and height of room, $h_{\mathrm{r}}$.

output $y$ at all, hence parameter $\theta$ can not be used for improving the model fit. On the other hand, if $\frac{\partial y}{\partial \theta} \neq 0$, this indicates that changing $\theta$ will change the output $y$ and allow for improved model fit. The transient change of $\frac{\partial y}{\partial \theta}$ is also informative wrt. what parts of the model parameter $\theta$ can be used to improve, e.g., steady state value, time constants, etc.

Both OMJulia-OpenModelica and the DifferentialEquation package for Julia support sensitivity computations. Here, we illustrate the results of sensitivity computations in OMJulia-OpenModelica for $y=$ $\left(T_{\mathrm{al}}, T_{\mathrm{pq}}, T_{\mathrm{r}}, T_{\mathrm{fb}}, T_{\mathrm{cb}}\right)$ and $\theta=\left(k_{\mathrm{pq}}, h_{\mathrm{r}}\right)$. In the sensitivity computations, we need to specify inputs. We start with inputs $u=\left(\begin{array}{ccccc}T_{\mathrm{a}}^{\mathrm{r}} & T_{\mathrm{a}}^{\mathrm{t}} & \dot{V}_{\ell} & u_{\mathrm{P}} & u_{\mathrm{v}}\end{array}\right)$ given by nominal values

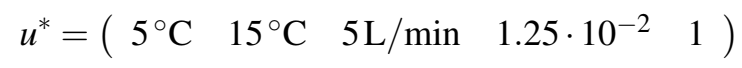

at $t=0$ and corresponding steady states, and inject a step change in $u_{\mathrm{v}}$ from $u_{\mathrm{v}}=u_{\mathrm{v}}^{*}=1$ to $u_{\mathrm{v}}=0.8$ at time $t=1 \mathrm{~h}$. Sensitivities of model $\mathrm{fhm}$ are computed numerically via OMJulia command

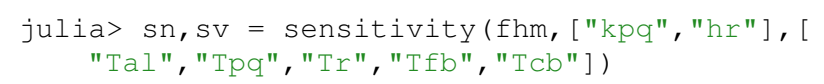

The results are displayed in Fig. 10.

To properly assess parameter identifiability, one needs to consider the rank of the sensitivity matrix, see, e.g., (Sarmiento Ferrero et al., 2006).

\subsection{Poles and zeros}

OMJulia, the Julia API to OpenModelica, allows for automatic differentiation for linearization of models. The algorithm does not handle the original buoyancy conduction 

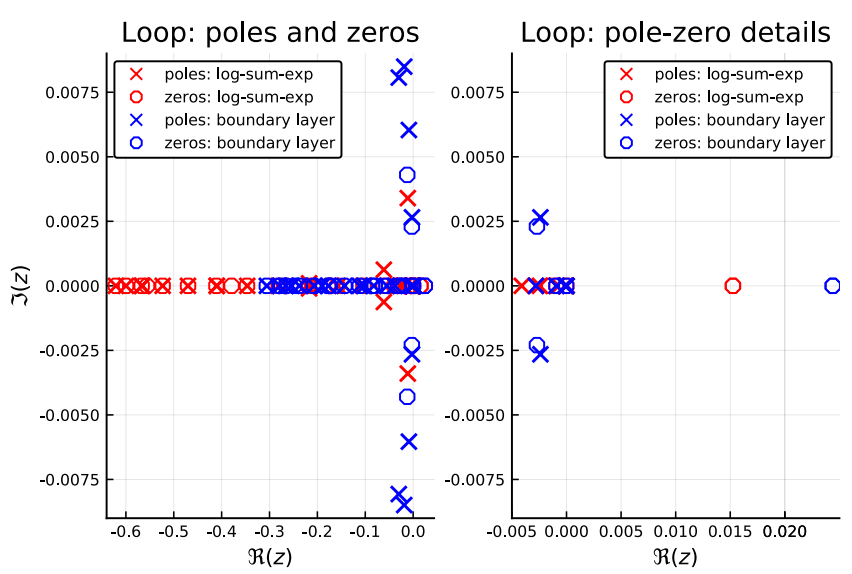

Figure 11. Poles marked with $\times$ (red: log-sum-exp approximation, blue: boundary layer approximation), and transmission zeros marked with $\circ$.

expression due to the non-differentiability of $k_{\mathrm{b}}$ at $\frac{\partial T}{\partial z}=0$, but works with the two approximate conductivity expressions. In this section, we consider inputs $u$ and outputs $y$ defined as:

$$
\begin{aligned}
& u=\left(\begin{array}{ccccc}
T_{\mathrm{a}}^{\mathrm{r}} & T_{\mathrm{a}}^{\mathrm{t}} & \dot{V}_{\ell} & u_{\mathrm{P}} & u_{\mathrm{v}}
\end{array}\right) \\
& y=\left(\begin{array}{cccc}
T_{\mathrm{i}} & T_{\mathrm{i}}^{\ell} & T_{\mathrm{pq}} & T_{\mathrm{r}}
\end{array}\right)
\end{aligned}
$$

with nominal input values

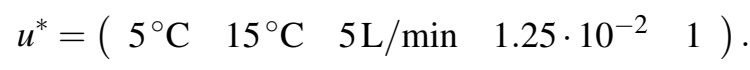

Linearization of model fhlse is carried out and piped ( $>$ ) into a Julia state space object sys_lse via OMJulia+Julia ControlSystems command

1 julia $>$ sys_lse $=$ linearize $($ fhlse $) \mid>x \rightarrow$ ss (

$$
x . .)
$$

When starting the model at steady state for the nominal inputs $u^{*}$, the combined heater + floor heating system gives a linear approximation with poles and transmission zeros as in Fig. 11.

As seen, there is some difference in both poles and transmission zeros of the two approximations. The right panel of Fig. 11 clearly indicates that both buoyancy approximations exhibit right-half-plane zeros in the complex plane, which indicates limitations on how fast the system can be controlled. These right-half-plane zeros are due to advection in the floor heating loop, and the Padé approximation that has been applied. It is difficult to see from Fig. 11 whether all poles of the system lie in the left-halfplane of the complex plane, but the simulations show that the system in fact is stable.

The precise location of transmission zeros gives additional information related to that of a possible inverse response found through step responses, see, e.g., (Lie, 1995). Observe that the two buoyancy conductivity approximations give different results wrt. poles and zeros. It is believed that the boundary layer approach is more accurate than that of the log-sum-exp approach.

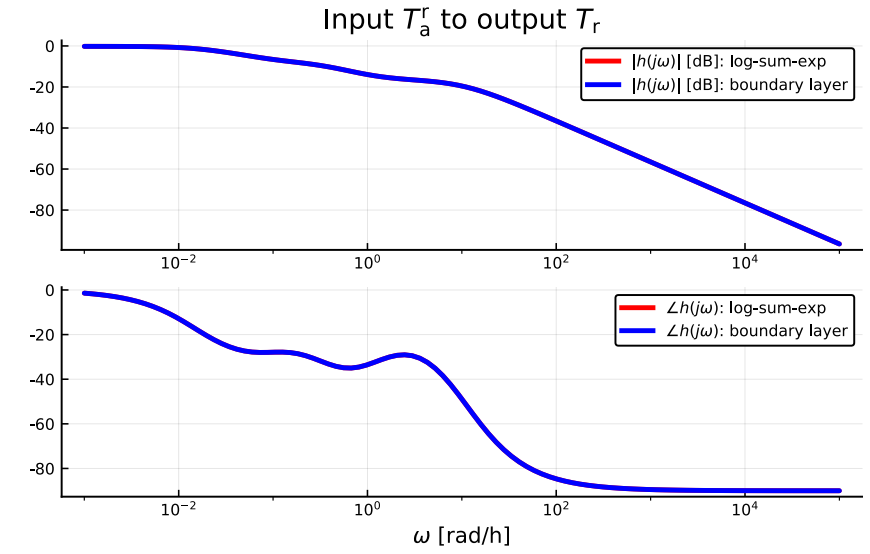

Figure 12. Bode plot of transfer function from outdoor temperature $T_{\mathrm{a}}^{\mathrm{r}}$ to room temperature $T_{\mathrm{r}}$.

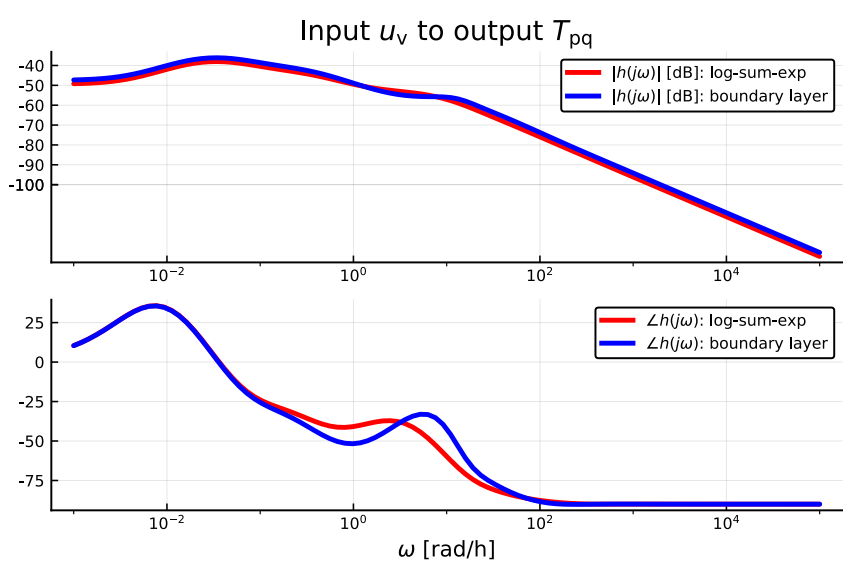

Figure 13. Bode plot of transfer function from heater split range valve signal $u_{\mathrm{v}}$ to parquet temperature $T_{\mathrm{pq}}$.

\subsection{Bode plots}

It is of interest to also compare the Bode plots from $u$ to $y$ of the linear approximations of the two buoyancy conduction approximations. In this case, we consider the following inputs $u$ and outputs $y$ :

$$
\begin{aligned}
& u=\left(\begin{array}{ccc}
T_{\mathrm{a}}^{\mathrm{r}} & \dot{V}_{\ell} & u_{\mathrm{v}}
\end{array}\right) \\
& y=\left(\begin{array}{ccc}
T_{\mathrm{i}}^{\ell} & T_{\mathrm{pq}} & T_{\mathrm{r}}
\end{array}\right) .
\end{aligned}
$$

The reason why we do not consider input $u_{\mathrm{P}}$ is that it turns out that the outputs are insensitive to $u_{\mathrm{P}}$. The following selected Bode plots are representative of the similarity of the two approximations. Transfer function $\frac{T_{\mathrm{r}}}{T_{\mathrm{a}}^{(}}(s)$, Fig. 12.

Observe that the two approximations are overlapping. Transfer function $\frac{T_{\mathrm{pq}}}{u_{\mathrm{v}}}(s)$, Fig. 13 .

Bode plots are standard tools for tuning SISO PID controllers. The two plots in Figs. 12-13 are representative of the variation in similarity between Bode plots from the boyancy conductivity approximations; for a real control design, the Bode plot from $u_{\mathrm{P}}$ to $T_{\mathrm{r}}$ would be more relevant, but controller design is not the main focus of this paper; rather the focus is on tools to analyze models. 


\section{Conclusions}

A model for floor heating of buildings is studied wrt. control relevant properties. The main emphasis is not on the analysis itself, but rather on the use of modern simulation tools to carry out such analysis. For a more realistic analysis, a better balanced system should be considered (size of heater vs. heat loss in room) with improved description of the living space (e.g., more rooms, thermal mass of furniture, ventilation, etc.).

The results indicate the importance of a suitable model description which is differentiable (stratification): this is important both for linearized analysis and controller design, but a proper choice of approximation will also allow for more flexibility in choice of differential equation solvers. The log-sum-exp approximation is locally unphysical, which probably also is the reason why this approximation requires very good solvers.

The results also indicate the ease at which control analysis can be carried out. Step responses are important for assessing overall open loop time constants, as well as indicating the presence of inverse response/"unstable" system zeros. Sensitivity analysis is important for assessing parameter identifiability. The location of open loop poles is related to open loop time constants, while system zeros give crucial information about how fast a closed loops system can be made. Finally, a Bode plot is often used for simple design of linear controllers.

In reality, temperature control in buildings is often done using thermostats and other nonlinear controllers. Still, it is of interest to consider how a more advanced controller - even a linear one - can be utilized for improved use of energy in building temperature control. The methods discussed in this paper constitute a first step in such a control design.

\section{References}

Karl Johan Åstrøm and Richard M. Murray. Feedback Systems. An Introduction for Scientists and Engineers. Princeton University Press, Princeton, NJ, 2008. ISBN 978-0-691-13576-2.

Ashish Bhattarai, Zahir Barahmand, Sina Orangi, and Bernt Lie. Extended model for control of thermal energy in buildings. In Submitted to SIMS 2020, 2020.

Peter Fritzson. Principles of Object-Oriented Modeling and Simulation with Modelica 3.3: A Cyber-Physical Approach. Wiley-IEEE Press, Piscataway, NJ, second edition, 2015. ISBN 978-1-118-85912-4.

N.S. Jayakumar, A. Agrawal, M.A. Hashim, and J.N. Sahu. Experimental and theoretical investigation of parametric sensitivity anddynamics of a continuous stirred tank reactor for acid catalyzedhydrolysis of acetic anhydride. Computers and Chemical Engineering, 35:1295-1303, 2011.

Casper Amandus Johansen, Bernt Lie, and Nils-Olav Skeie. Models for control of thermal energy in buildings. In Erik Dahlquist, Esko Juuso, Bernt Lie, and Lars Eriksson, editors,
Proceedings of the 60th Conference on Simulation and Modelling (SIMS 59), number 170 in Linköping Electronic Conference Proceedings. Linköping University Electronic Press, 2019. doi:https://doi.org/10.3384/ecp20170.

Huibert Kwakernaak and Raphael Sivan. Linear Optimal Control Systems. Wiley-Interscience, New York, 1972.

Jesus Lago, Fjo De Ridder, Wiet Mazairac, and Bart De Schutter. A 1-dimensional continuous and smooth model for thermally stratified storage tanks including mixing and buoyancy. Applied Energy, 248:640-655, 2019.

Bernt Lie. Methods of Model Based Process Control, chapter Attainable Performance in LQG Control, pages 263-295. NATO ASI Series, Series E: Applied Sciences - Vol. 293. Kluwer Academic Publishers, Dordrecht, 1995. Proceedings of the NATO Advanced Study Institute on Methods of Model Based Process Control, Antalya, Turkey, August 7-17, 1994.

Bernt Lie, Carlos Pfeiffer, Nils-Olav Skeie, and Hans-Georg Beyer. Models for solar heating of buildings. In Alireza Rezania Kolai, Kim Sørensen, and Mads Pagh Nielsen, editors, Proceedings, 55th International Conference of Scandinavian Simulation Society, pages 28-38, Aalborg University, Denmark, October 2014. Scandinavian Simulation Society, Linköping University Electronic Press. doi:www.ep.liu.se/ecp/108/ecp14108.pdf. October 21-22 2014.

Bernt Lie, Arunkumar Palanisamy, Alachew Mengist, Lena Buffoni, Martin Sjölund, Adeel Asghar, Adrian Pop, and Peter Fritzson. OMJulia: An OpenModelica API for JuliaModelica Interaction. In Proceedings of the 13th International Modelica Conference, pages 699-708, February 2019. doi:10.3384/ecp19157. Regensburg, Germany, March 4-6, 2019.

Christopher Rackauckas and Qing Nie. DifferentialEquations.jl - A Performant and Feature-Rich Ecosystem for Solving Differential Equations in Julia. Journal of Open Research Software, 5(15), 2017. doi:10.5334/jors.151.

Cristina Sarmiento Ferrero, Qian Chai, Marta Dueñas Díez, Sverre H. Amrani, and Bernt Lie. Systematic analysis of parameter identifiability for improved fitting of a biological wastewater model to experimental data. Modeling, Identification and Control, 27(4):219-238, 2006. doi:10.4173/mic.2006.4.2.

Arvind Varma, M. Morbidelli, and H. Whu. Parametric Sensitivity in Chemical Systems. Cambridge University Press, 1999. ISBN 978-0521621717.

R. Viskanta, M. Behnia, and A. Karalis. Interferometric observations of the temperature structure in water cooled or heated from above. Advances in Water Resources, 1(2):57-69, 1977.

Evangelos Vrettos. Control of Residential and Commercial Loads for Power System Ancillary Services. PhD thesis, ETH, Power Systems Laboratory, 2016.

Zhijie Xu, Ruisheng Diao, Shuai Lu, Jianming Lian, and Yu Zhang. Modeling of electric water heaters for demand response: A baseline pde model. IEEE Transactions on Smart Grid, 5(5):2203-2210, September 2014. doi:10.1109/TSG.2014.2317149. 